\title{
Identification of key miRNA-mRNA pairs in septic mice by bioinformatics analysis
}

\author{
JIANXIN CHEN ${ }^{1,2}$, MIN LIN ${ }^{3}$ and SEN ZHANG ${ }^{1}$ \\ ${ }^{1}$ Department of Colorectal Surgery, The First Affiliated Hospital of Guangxi Medical University, Nanning, Guangxi 530021; \\ ${ }^{2}$ The First Department of Gastrointestinal Surgery, The Affiliated Hospital of Putian University; \\ ${ }^{3}$ School of Information Engineering, Putian University, Putian, Fujian 351100, P.R. China
}

Received April 11, 2019; Accepted July 26, 2019

DOI: $10.3892 / \mathrm{mmr} .2019 .10594$

\begin{abstract}
Sepsis is one of the most common causes of death among critically ill patients in intensive care units worldwide; however, the microRNAs (miRNAs/miRs) involved in the sepsis process (and their target genes) are largely unknown. The present study integrated miRNA and mRNA datasets to elucidate key sepsis-related miRNA-mRNA pairs. The datasets, GSE74952 and GSE55238 were downloaded from the Gene Expression Omnibus. By performing bioinformatics analysis such as GEO2R, miRNA target gene prediction, Gene Ontology analysis, Kyoto Encyclopedia of Genes and Genomes pathway analysis and miRNA-mRNA network analysis, a total of four sepsis-related miRNA-mRNA pairs were successfully obtained. Mmu-miR-370-3p, cluster of differentiation (CD)8a, CD247, Zap70 and inhibitor of nuclear factor $\kappa$ B kinase subunit $\beta$ (Ikbkb) were identified as the components involved in these pairs, and these genes were enriched in the T-cell receptor signaling pathway. Finally, reverse transcription-quantitative PCR results validated that the expression levels of the four genes (CD8a, CD247, Zap70 and Ikbkb) in the sepsis model mice were consistent with the microarray analysis. In conclusion, the present study identified four sepsis-related miRNA-mRNA pairs using bioinformatics analysis. These results indicated that the candidate miRNA-mRNA pairs may be involved in the regulation of immunity in sepsis, which may in turn act as indicators or therapeutic targets for sepsis.
\end{abstract}

Correspondence to: Dr Sen Zhang, Department of Colorectal Surgery, The First Affiliated Hospital of Guangxi Medical University, 6 Shuangyong Road, Nanning, Guangxi 530021, P.R. China

E-mail: 2469240843@qq.com

Abbreviations: miRNA, microRNA; DEGs, differentially expressed genes; DAVID, Database for Annotation, Visualization and Integration Discovery; GEO, Gene Expression Omnibus; CLP, cecal ligation and puncture; GO, Gene Ontology; KEGG, Kyoto Encyclopedia of Genes and Genomes; FC, fold-change; TCR, T-cell receptor

Key words: sepsis, GEO, bioinformatic analysis, differentially expressed genes, mRNA, miRNA

\section{Introduction}

Sepsis is defined as a life-threatening organ dysfunction caused by a dysregulated host response to an infection (1). Sepsis is particularly lethal as it often follows a linear continuum from systemic inflammatory response syndrome through to septic shock and organ function failure (2-4). Despite advancements in antibiotic therapy, immunotherapy and resuscitative strategies, sepsis remains the leading cause of death in intensive care units (5).

In the pathophysiological process of sepsis, certain gene expression levels markedly change in vivo and contribute to the outcome of the disease $(6,7)$. Gene microarray or gene profiling are molecular detection techniques that have been used for a number of years. Gene microarray analysis can quickly detect gene expression levels at various time-points, which is particularly effective for the screening of differentially expressed genes (DEGs).

MicroRNAs (miRNAs/miRs) are a group of small non-coding RNAs that regulate gene expression at the post-transcriptional level and serve as key regulators in the progression of many types of disease $(8,9)$. To investigate the roles of miRNAs and their target genes in sepsis, Chen et al (10) detected the altered expression of specific miRNAs and their target genes in patients with sepsis, and identified specific miRNA and target genes involved in the activation of immune and inflammatory responses. In addition, previous studies have also indicated that the expression level of miRNA was correlated with the mortality of sepsis patients and certain miRNAs in the blood can be used to predict the prognosis of sepsis $(11,12)$. The aim of the present study was to obtain key sepsis-related miRNA-mRNA pairs in mice by analyzing mRNA and miRNA microarray datasets, in order to provide molecular targets for diagnostic or therapeutic strategies.

\section{Materials and methods}

Microarray data. The sepsis and control group blood gene expression profiles GSE74952 and GSE55238 in mice were downloaded from the Gene Expression Omnibus (GEO) database of the National Center for Biotechnology Information (www.ncbi.nlm.nih.gov/geo). The microarray data of 
GSE74952 was based on GPL21136 Platforms (Multiplex Circulating miRNA Assay) and the miRNA profiles of 5 sepsis samples and four control samples were obtained. These 5 sepsis samples were obtained from septic mice models that were created by cecal ligation and puncture (CLP). The microarray data of GSE55238 was based on GPL1261 Platforms (Affymetrix Mouse Genome 4302.0 Array) and the mRNAs profile of 4 sepsis samples and 4 control samples were obtained. These 4 sepsis samples were also obtained from CLP septic mice models. The present study chose these 4 sepsis samples as Day 1, which was the same time-point used in GSE74952. GSE74952 was conducted at the Massachusetts General Hospital, Charlestown, USA; GSE55238 was conducted at the University of Florida, Gainesvile, USA.

Data preprocessing and screening strategy. A multi-step strategy (Fig. 1) was used to identify genes dysregulated in the sepsis model mice relative to the control group. First, GEO2R was applied to identify the differentially expressed miRNAs and mRNAs between sepsis and control samples. GEO2R (www.ncbi.nlm.nih.gov/geo/geo2r/) is an interactive web tool that is widely applied to detect DEGs by comparing two groups of samples in a GEO series (11). An adjusted $\mathrm{P}<0.05$ and $\mid \log 2$ fold-change (FC) $\mid>1$ were set as the cut-off criteria.

Second, the TargetScan database (version 7.2; www. targetscan.org/mmu-72/) was used to predict the target genes of dysregulated miRNAs and the overlap between the negatively correlated target genes and differentially expressed mRNAs was established. Gene sets data was processed using VENNY 2.1 software (bioinfogp.cnb.csic.es/tools/venny/index.html).

Third, these interacting genes were classified according to Gene Ontology (GO; http://geneontology.org/) and Kyoto Encyclopedia of Genes and Genomes (KEGG; www.genome. jp/kegg) (13) pathways using Database for Annotation, Visualization and Integration Discovery (DAVID) software (david.ncifcrf.gov/). Based on the enriched GO terms and significant KEGG pathways, the sepsis-related mRNAs were screened.

Finally, Cytoscape software (version 3.6.0; https://cytoscape.org/) was used to construct a miRNA-mRNA network. Based on the sepsis-related mRNAs, the sepsis-related miRNA-mRNA pairs were obtained.

Validation by reverse transcription-quantitative $(R T-q) P C R$ in mouse sepsis models. A total of $12 \mathrm{C} 57 \mathrm{BL} / 6$ male mice (aged 8-10 weeks, weighing 20-25 g) were purchased from the Experimental Animal Center of Guangxi Medical University. All mice were housed in specific pathogen-free facilities and acclimated for 1 week before the operation. Mice were maintained at $22^{\circ} \mathrm{C}$ with $55 \%$ humidity under a $12: 12$-h light/dark cycle for 1 week, with access to food and water ad libitum. A total of 6 mice were employed to establish the sepsis model using the CLP procedure according to a previously reported method (12). The remaining 6 mice were sham-operated to produce the control group; control mice underwent the same procedure as the sepsis model mice but without ligation and puncture of the cecum. All operation procedures were under sterile conditions. After 1 day, $\sim 1 \mathrm{ml}$ blood was collected from the hearts of the mice. Red blood cells were lysed using BD Pharm Lyse ${ }^{\mathrm{TM}}$ lysing buffer (Becton, Dickinson and Company).

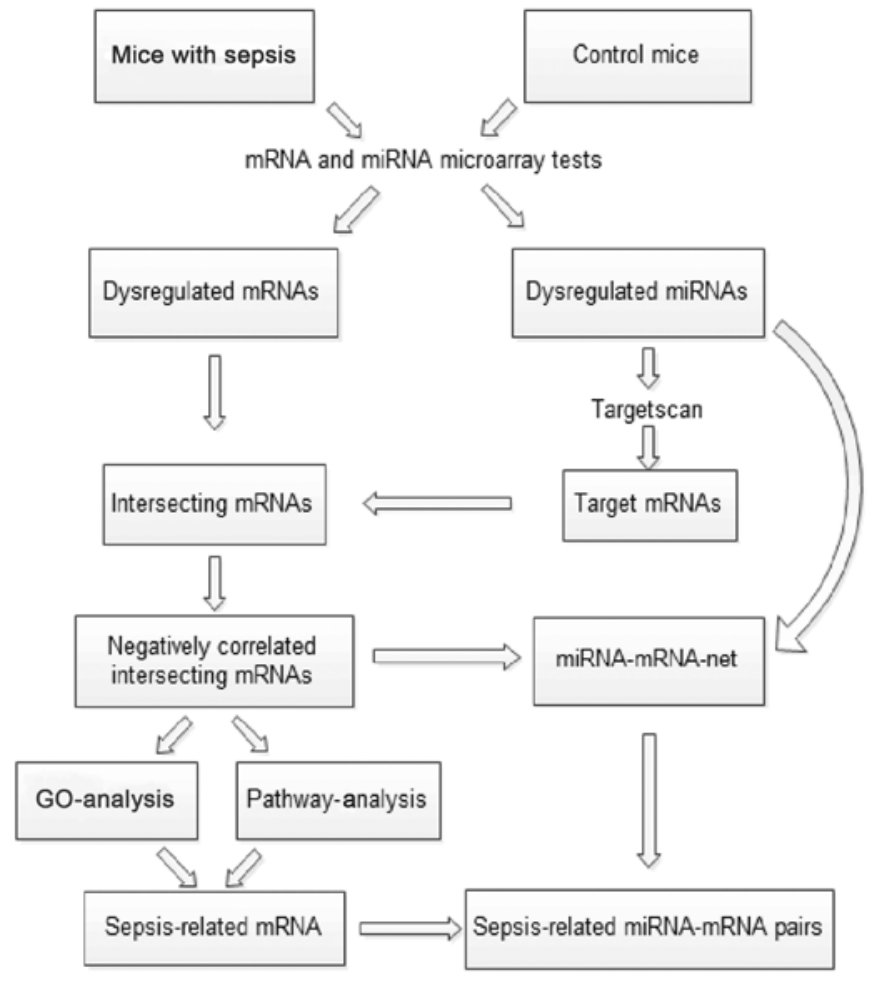

Figure 1. The multi-step strategy used in the present study. GO, gene ontology; miRNA, microRNA.

Total RNA was extracted using RNAiso Plus (Takara Bio, Inc.) and then reverse transcribed into cDNA using PrimeScript ${ }^{\mathrm{TM}}$ RT reagent kit with gDNA Eraser (Takara Bio, Inc.) at $42^{\circ} \mathrm{C}$ for $2 \mathrm{~min}, 37^{\circ} \mathrm{C}$ for $15 \mathrm{~min}$ and $85^{\circ} \mathrm{C}$ for $5 \mathrm{sec}$ according to the manufacturer's protocol. qPCR was conducted using the SYBR Premix Ex Taq ${ }^{\mathrm{TM}}$ II (Takara Bio, Inc.) according to the manufacturer's protocol. The PCR primers were designed by the National Center for Biotechnology Information (www. ncbi.nlm.nih.gov/) and provided by Sangon Biotech Co., Ltd., (Table I). GAPDH was selected as the internal reference for the genes. RT-qPCR thermocycling conditions included: Pre-denaturation step at $95^{\circ} \mathrm{C}$ for $30 \mathrm{sec}$, followed by 40 cycles of denaturing at $95^{\circ} \mathrm{C}$ for $5 \mathrm{sec}$ and extension at $60^{\circ} \mathrm{C}$ for $34 \mathrm{sec}$ (Applied Biosystems 7500 Real Time PCR System); each sample was performed in triplicate. Relative expression values were calculated using the $2^{-\Delta \Delta C q}$ method (14). The present study was approved by the Animal Care Committee of Guangxi Medical University (approval no. 201904001).

Statistical analysis. Data were analyzed using SPSS version 17.0 (SPSS, Inc.). Continuous variables are presented as the mean \pm standard deviation. A Student's t-test was applied for comparisons between two groups. If the variance was not equal between two groups, a Mann-Whitney U test was used for statistical analysis. $\mathrm{P}<0.05$ was considered to indicate a statistically significant difference. In addition to P-value, the false discovery rate value was also used to rank the significance of the pathways.

\section{Results}

Identification of differentially expressed miRNAs and mRNAs in sepsis model mice. The gene expression levels of miRNAs 
Table I. Primer sequences for reverse transcription-quantitative PCR.

\begin{tabular}{lll}
\hline Gene & \multicolumn{1}{c}{ Forward primer (5'-3') } & \multicolumn{1}{c}{ Reverse primer $\left(5^{\prime}-3^{\prime}\right)$} \\
\hline CD8a & TTCTGTCGTGCCAGTCCTTC & TGGGACATTTGCAAACACGC \\
CD247 & ATGGGTATTGACTCGCTCCG & CAGGCTTCACCACTGAAATAAG \\
Zap70 & ATCATGGCTTATGGCCGTGT & CATGCACTCCCGGGTTAGAG \\
Ikbkb & CCCACCCCTCCTCTCCTTAC & TTCATACTGCCTCTGCGGTG \\
GAPDH & CCCTTAAGAGGGATGCTGCC & ACTGTGCCGTTGAATTTGCC \\
\hline
\end{tabular}

$\mathrm{CD}$, cluster of differentiation.

Table II. Significantly dysregulated miRNAs when comparing sepsis and control mice.

\begin{tabular}{lrrr}
\hline miRNA & $\begin{array}{c}\text { Adjust } \\
\text { P-value }\end{array}$ & LogFC & $\begin{array}{c}\text { Differential } \\
\text { expression }\end{array}$ \\
\hline miR-155-5p & $<0.001$ & 2.911 & Upregulated \\
miR-146a-5p & 0.004 & 2.527 & Upregulated \\
miR-21-5p & $<0.001$ & 2.337 & Upregulated \\
miR-370-3p & 0.001 & 2.234 & Upregulated \\
miR-28-5p & 0.001 & 2.231 & Upregulated \\
miR-590-5p & 0.011 & 1.879 & Upregulated \\
miR-29a-3p & 0.011 & 1.855 & Upregulated \\
miR-19a-3p & $<0.001$ & 1.731 & Upregulated \\
miR-103a-3p & 0.031 & 1.349 & Upregulated \\
let-7i-5p & 0.031 & 1.049 & Upregulated \\
\hline
\end{tabular}

miRNAs/miRs, microRNAs.

and mRNAs were download from the GEO database, respectively. Based on GEO2R analysis, a total of 63 differentially expressed miRNAs and a total of 21,765 differentially expressed mRNAs were identified in septic mice compared with the control samples. There were 7,508 upregulated and 14,257 downregulated mRNAs in the GSE55238 dataset (Fig. 2A), and 44 up- and 19 downregulated miRNAs in the GSE74952 dataset (Fig. 2B). Screened with the cut-off criteria of adjusted $\mathrm{P}<0.05$ and $\log 2 \mathrm{FCl}>1,10$ differentially expressed miRNAs (Table II) and 3,724 differentially expressed mRNAs were identified in sepsis model mice compared with the control mice. These candidate targets included 10 upregulated miRNAs and 3,293 up- and 431 downregulated mRNAs.

miRNA target gene prediction. Target mRNAs for differentially expressed miRNAs were predicted using the TargetScan database. A total of 7,043 target genes were successfully predicted in 6 miRNAs, including mmu-miR-155-5p, mmu-miR-146a-5p, mmu-miR-370-3p, mmu-miR-29a-3p, mmu-miR-19a-3p and mmu-let-7i-5p.

Selection of disease-associated miRNA-mRNA pairs. A total of 1,510 intersecting mRNAs between the predicted target mRNAs and differentially expressed mRNAs were selected
(Fig. 2C), of which $126 \mathrm{mRNAs}$ that were negatively correlated with their predicted miRNAs matches were selected (Fig. 2D). Based on differentially expressed miRNAs and their negatively correlated mRNAs, a total of 147 miRNA-mRNA pairs were predicted.

Selected mRNA ontology analysis in sepsis model mice. The functional enrichment of 126 candidate DEGs were analyzed using DAVID (david.ncifcrf.gov/; Table III). A total of three GO category results are presented including biological processes, cellular components and molecular functions. The biological process results revealed that the selected DEGs were mainly enriched in 'cellular response to cAMP', 'actin cytoskeleton organization' and 'calcium ion regulated exocytosis' (Fig. 3A). The cellular component results revealed that selected DEGs were mainly enriched in 'cytoplasm', 'membrane' and 'membrane raft' (Fig. 3B). The molecular function analysis revealed that the selected DEGs mainly enriched in 'protein binding', 'phospholipid binding' and 'actin binding' (Fig. 3C). These results demonstrated that the majority of the selected DEGs were enriched in 'binding' and 'cell signaling'.

Signaling pathway enrichment analysis. The signaling pathway enrichment of 126 candidate DEGs were analyzed using KEGG pathway online databases. The selected DEGs were significantly enriched in 'T-cell receptor signaling pathway', 'tryptophan metabolism' and 'proteoglycans in cancer' (Fig. 3D). Based on a previous study (15), the T-cell receptor signaling pathway is an important signaling pathway associated with sepsis. In the present study, 4 sepsis-related mRNAs were found in this pathway including CD8a, CD247, Zap70 and inhibitor of nuclear factor $\kappa$ B kinase subunit $\beta$ (Ikbkb; Table IV; Fig. 4).

miRNA-mRNA network construction. A total of 126 candidate mRNAs and 6 miRNAs were analyzed using Cytoscape software. A total of 147 miRNA-mRNA pairs were presented in the network. Based on this, 4 sepsis-related mRNAs and 4 sepsis-related miRNA-mRNA pairs were obtained (Fig. 5).

$R T$-qPCR validation. To confirm the reliability of the findings from the bioinformatics microarray analysis, 4 mRNAs [cluster of differentiation (CD)8a, CD247, Zap70 and Ikbkb] were selected for validation by RT-qPCR in 6 pairs of matched sepsis and control C57BL/6 mice. According to 


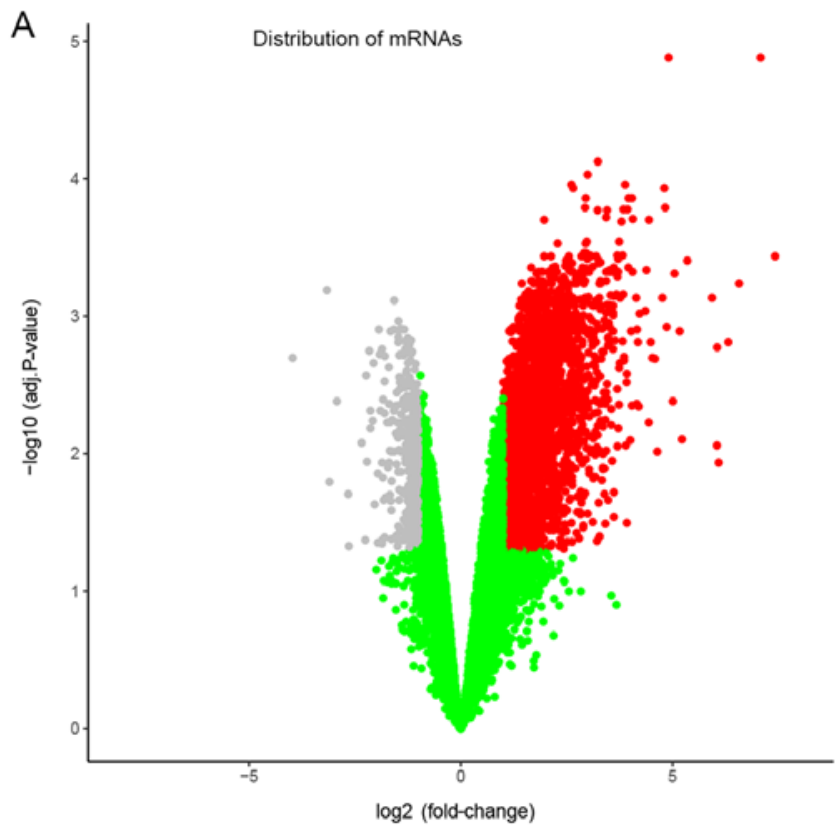

C Intersecting mRNAs between predicted target mRNAs (a) and DEGs (b)

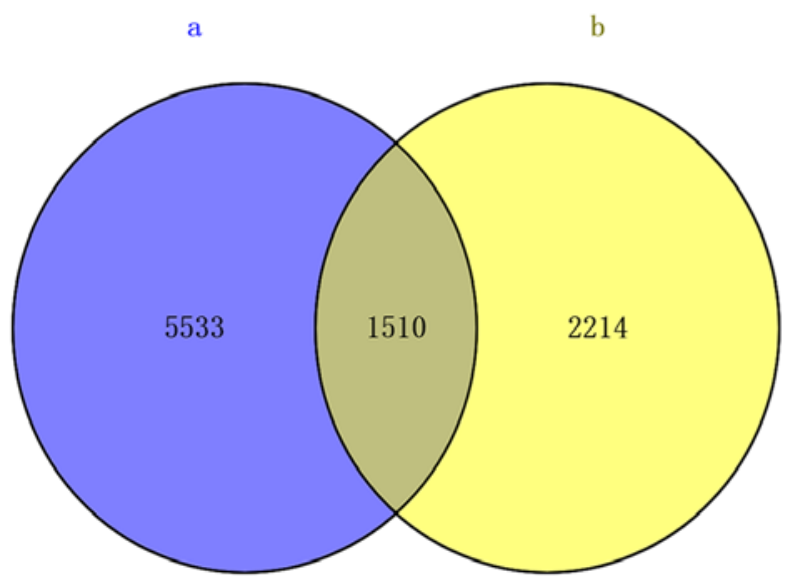

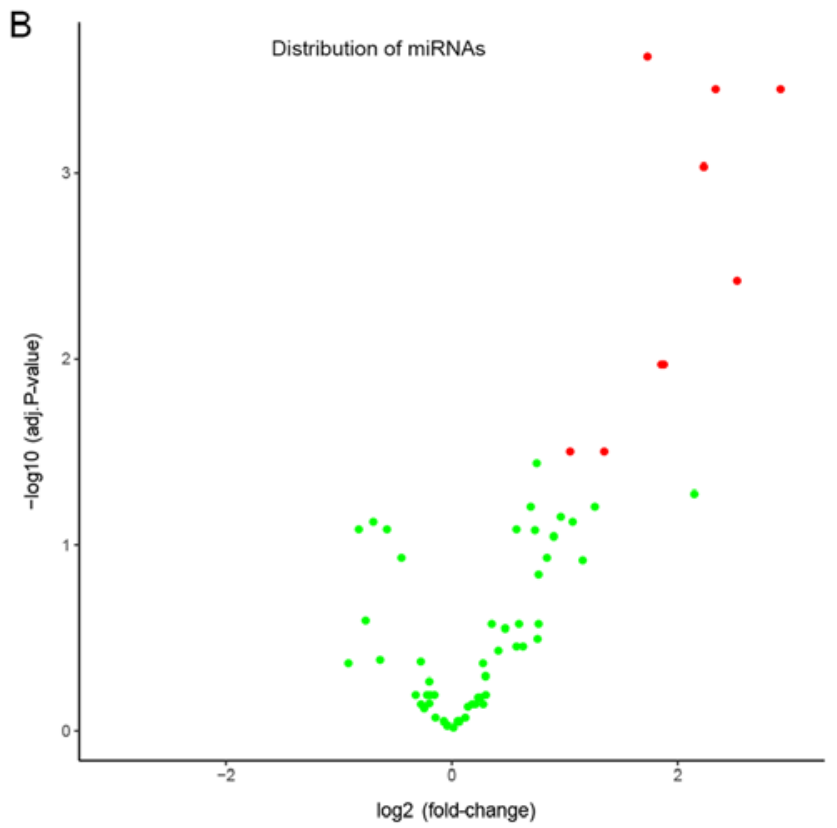

D Overlap between intersecting mRNAs (c) and negatively correlated mRNAs (d)

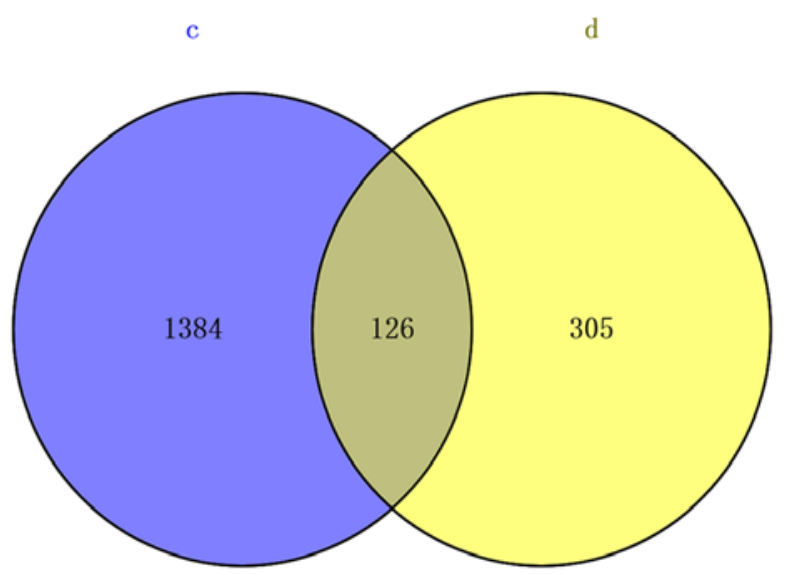

Figure 2. Two volcano plots of two GEO datasets and two Venn diagrams from the DEG screening. (A) The volcano plots of the mRNA and (B) miRNA datasets. (C) The intersecting mRNAs between the predicted target mRNAs and differentially expressed mRNAs. (D) The overlap between the intersecting mRNAs and the negatively correlated mRNAs. GEO, Gene Expression Omnibus; DEG, differentially expressed gene; miRNA, microRNA.

the experimental results, the relative expression levels were similar between microarray and RT-qPCR experiments. In sepsis model mice, the expression level of CD8a, CD247, Zap70 and Ikbkb were significantly downregulated $(\mathrm{P}<0.05$; Fig. 6).

\section{Discussion}

Numerous studies have been performed to reveal the underlying mechanisms of sepsis formation and progression in the past several decades; however, the mortality rates of sepsis are still very high worldwide $(3,5)$. The majority of previous studies have indicated that the high mortality of sepsis is correlated with immune dysfunction, which may be due to T-cell dysfunction $(16,17)$. However, which molecules change and how they interact during sepsis are still unclear. The present study integrated two datasets including miRNA and
mRNA microarrays from different groups, using bioinformatics methods to analyze data. Through DEG screening, miRNA gene target prediction, GO terms and KEGG pathway analysis, 1 sepsis-related miRNA and 4 sepsis-related mRNAs including mmu-miR-370-3p, CD8a, CD247, Zap70 and Ikbkb were obtained, of which mmu-miR-370 was upregulated and the others were downregulated. Subsequent RT-qPCR analysis validated this finding. Through the construction of the miRNA-mRNA network, 4 sepsis-related miRNA-mRNA pairs were identified.

A septic event triggers high levels apoptosis in immune cells such as T-cells, resulting in suppressed immune functions, often inducing an increased susceptibility to secondary infections $(5,15,18,19)$. The CLP mouse model of sepsis is considered the gold standard in experimental sepsis research. However, sepsis induction varies among different research groups, making it a challenge to compare data among different 
A

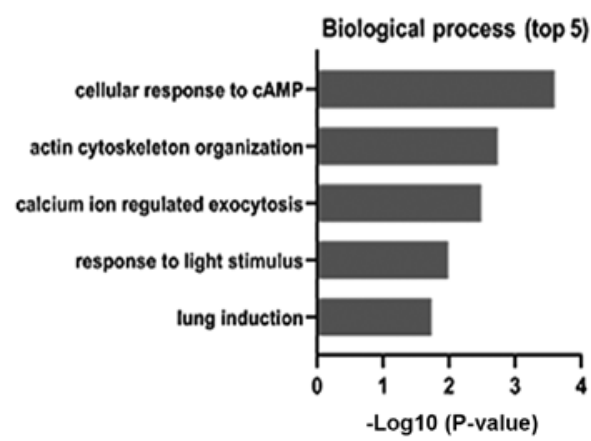

B
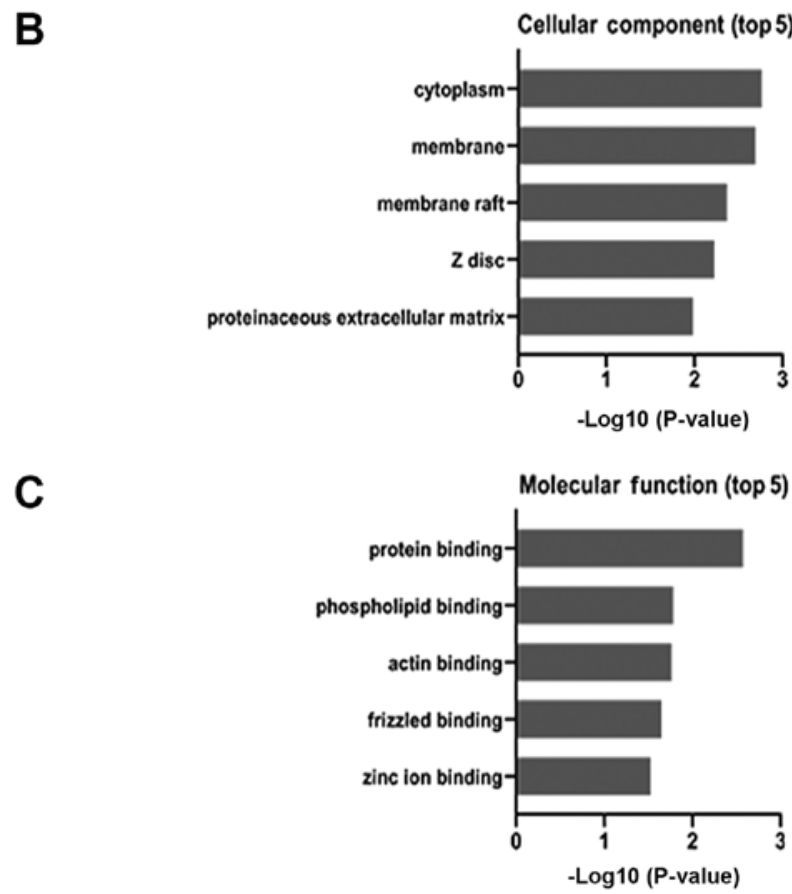

D

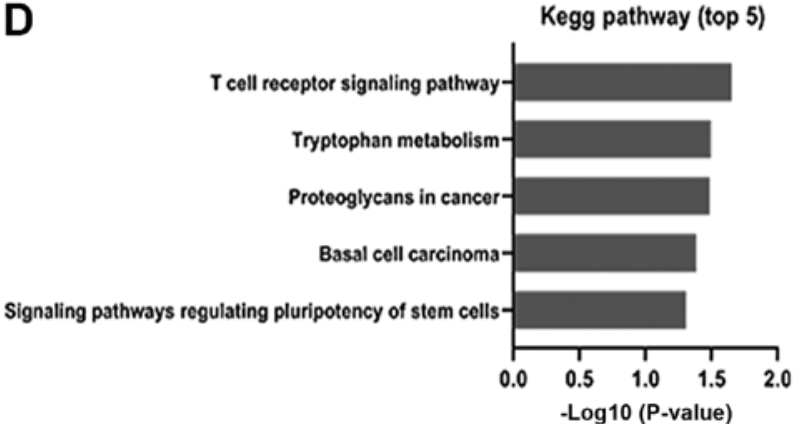

Figure 3. Top 5 results of GO enrichment and KEGG pathway analysis (A) Biological processes, (B) Cellular components and (C) Molecular functions. (D) KEGG pathway analysis. GO, Gene Ontology; KEGG, Kyoto Encyclopedia of Genes and Genome.

studies. Despite the variation in sepsis induction, the majority of studies have demonstrated that sepsis leads to a significant decrease in immune factors. For instance, Condotta et al (20) demonstrated that polymicrobial sepsis exacerbated $\mathrm{CD}^{+}$ T-cell exhaustion and reduced interferon- $\gamma$ production. In addition, Hotchkiss et al (21) indicated that there is a significant loss of B and CD4 T-cells in sepsis, which may contribute to immunosuppression.

In the pathological progression of sepsis, the host response is disturbed by excessive inflammation and immune suppression,
Table III. The 126 negatively correlated intersecting genes with their predicted miRNAs.

Style Genes name

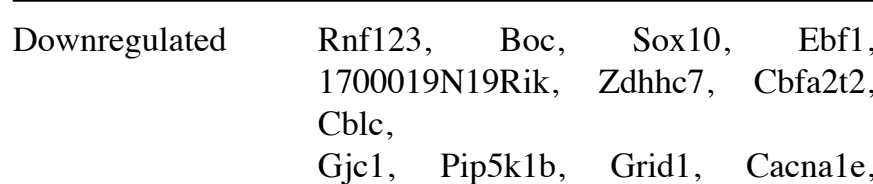
Arhgap17, Hip1r, Wnt2b, Fbln5, Cend1, Cd8a, Samd11, Trp53i11, Haao, Phxr4, Aanat, Ralgps2, Gulp1, P2rx3, Mlxipl, Hapln4, Naa40, Kcnk5, Ptgis, Fkbp8, Cgnl1, Dusp9, Fads1, R3hdm4, Apobec2, Clip4, Spsb1, Tet3, Arhgef18, Ikbkb, Myole, Specc1, Npepl1, Capn8, Polg, Klhl17, Nr5a1, 6330408A02Rik, Stx1a, Kmt2a, Il5ra, Wnt7a, Lurap1, Brat1, Marf1, Lynx1, Pde11a, H2-Eb2, Dcun1d2, Rnf165, Gpr50, Wnt2, Slc4a1, Ctnnd1, Wsb2, Zfp882, Dgcr6, Faim2, Synpo21, Fam222a, Hmg20a, Mark4, Bmp1, Bgn, Efna4, Unc45a, Ccr6, Bcl2, Tgif2, Cmtr2, Smtnl2, Slc8a1, Asphd2, Tmem86b, Eef2k, Ermp1, Ucp2, Adal, Snrnp70, Sumo3, Fam193a, 2-Mar, Atp9a, Dmtn, Dtnb, Gorasp1, Vps13a, Kynu, Usp15, Ank1, Pmf1, Slc2a8, Snap47, Cers4, Serpinh1, Mpp3, Plk2, Zap70, Igfbp5, Cd247, Akap4, Trappc12, Prrt4, Enpp2, Slc6a2, Ehd2, Wasf3, Psd, Rapgef4, Usp14, Slc26a7, Sgip1, K1, Adipor1

miRNAs, microRNAs.

and fails to return to normal homeostasis (22). The T-cell receptor signaling pathway is an important pathway involved in immune responses. Previous studies have suggested that the impairment of the T-cell receptor signaling pathway was one of important factors for immunosuppression in sepsis $(23,24)$.

Based on KEGG pathway analysis, the hub genes including CD8a, CD247, Zap70 and Ikbkb were enriched in the T-cell receptor signaling pathway. These genes play an important role in immunity to fight against foreign pathogens. CD8a encodes the CD8 $\alpha$ chain of the dimeric CD8 protein. CD8 is a coreceptor for the T-cell receptor (TCR)-mediated recognition of major histocompatibility complex class I, which involved cytotoxic $\mathrm{T}$ lymphocyte activation and target cell lysis (25). Therefore, $\mathrm{CD}^{+} \mathrm{T}$-cells play an important role in the control and eradication of invading pathogens (26). CD8 deficiency increases susceptibility to infection (27). Harland et al (28) revealed that $\mathrm{CpG}$ methylation of the CD8a locus has a potential role in the downregulation of CD8. However, whether this epigenetic modification is involved in the process of sepsis is still unclear. CD247 (also known as CD3z) is a part of the TCR complex, which plays a key role in receptor expression and signaling associated to T-cell 
Table IV. The top 5 signaling pathways of differentially expressed genes.

\begin{tabular}{llllll}
\hline KEGG pathway & \multicolumn{1}{c}{ Name } & Count & P-value & FDR & Genes \\
\hline mmu04660 & T-cell receptor signaling pathway & 4 & 0.022 & 22.085 & CD8a, CD247, Zap70, Ikbkb \\
mmu00380 & Tryptophan metabolism & 3 & 0.032 & 30.270 & Kynu, Haao, Aanat \\
mmu05205 & Proteoglycans in cancer & 5 & 0.032 & 30.696 & Wnt2, Cblc, Ank1, Wnt7a, Wnt2b \\
mmu05217 & Basal cell carcinoma & 3 & 0.041 & 37.321 & Wnt2, Wnt7a, Wnt2b \\
mmu04550 & Signaling pathways regulating & 4 & 0.049 & 42.819 & Wnt2, Dusp9, Wnt7a, Wnt2b \\
& pluripotency of stem cells & & & &
\end{tabular}

KEGG, Kyoto Encyclopedia of Genes and Genomes; FDR, false discovery rate; CD, cluster of differentiation.

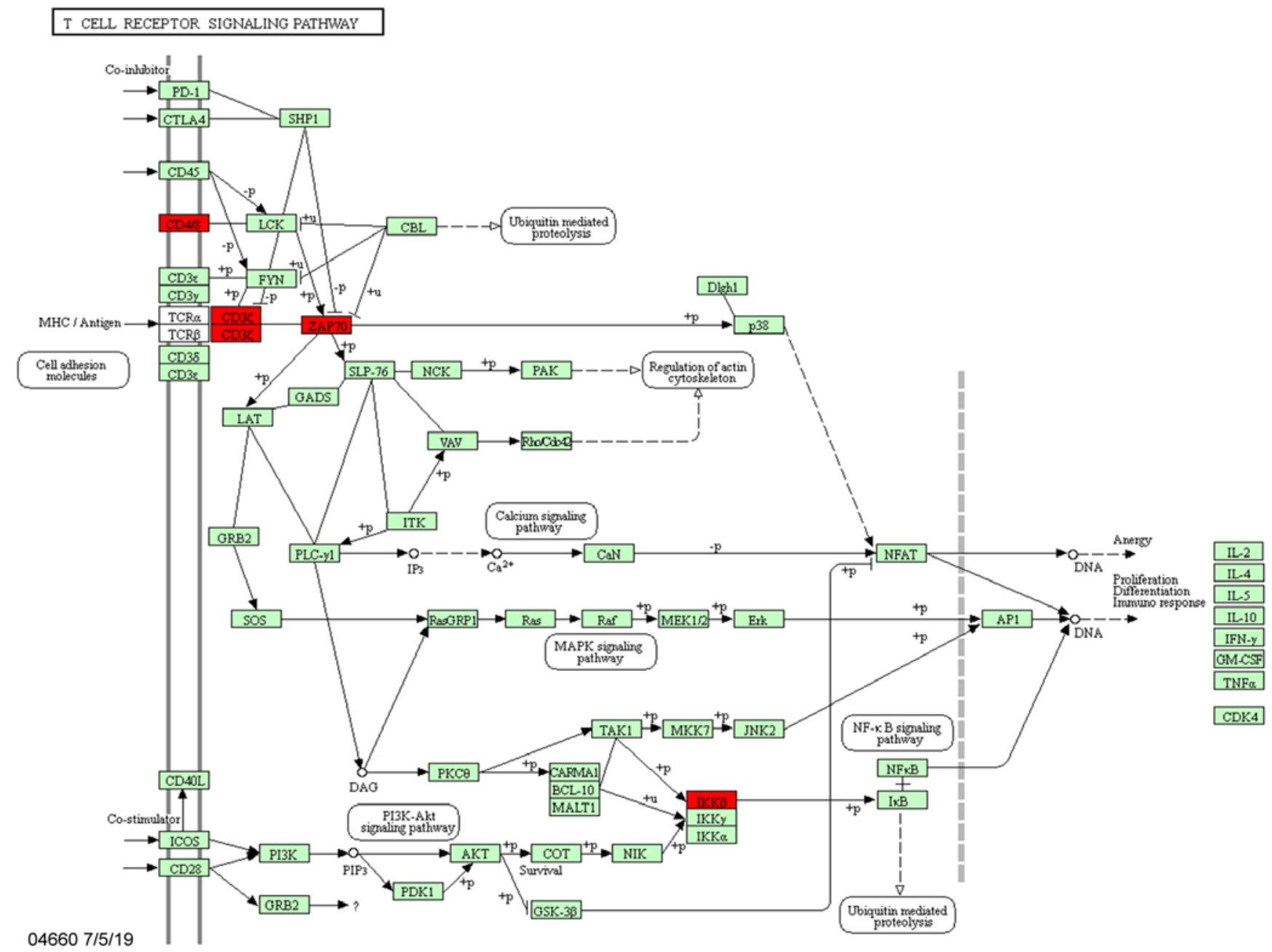

(c) Kanehisa Laboratories

Figure 4. T-cell receptor signaling pathway. Red regions represent the location of four sepsis-related mRNAs. Pathway map from the Kyoto Encyclopedia of Genes and Genomes published with permission from the copyright owner (13).

functions (29,30). Eldor et al (31) reported that CD247 downregulation is associated with immunosuppression in T-cells and was correlated with disease progression and severity in patients with type 2 diabetes. Zap70 was enriched in the TCR signaling pathway. The functional deletion of Zap70 can lead to a serious immunodeficiency (32). In these patients, T-cells are inadequately supervised by the thymus microenvironment, exhibit decreased apoptosis and cannot differentiate into Th2 T-cells (33). Huang et al (34) reported that the downregulation of Zap70 accelerated disease progression of neonatal sepsis. IKBKB encodes IKB kinase 2 (also known as IKK $\beta$ /IKK2), a component of the nuclear factor- $\kappa \mathrm{B}$ signaling pathway. IKK $\beta$ can phosphorylate and degrade the inhibitors of $\kappa \mathrm{B}$, and then improve target gene transcription $(35,36)$. A number of studies 


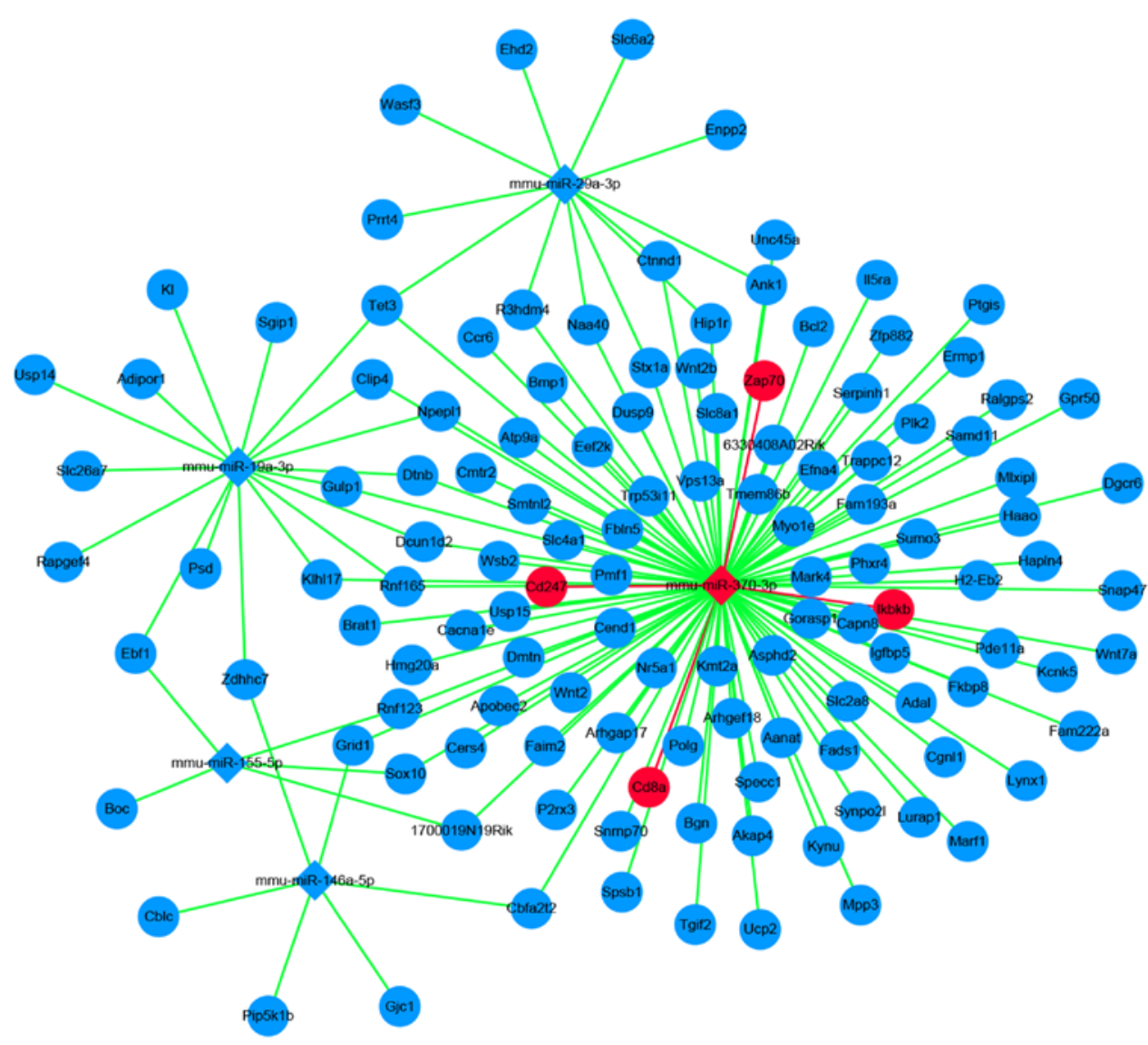

Figure 5. miRNA-mRNA-network. Diamonds represent miRNAs; circles represent mRNA; red nodes and edges represent sepsis-related miRNA-mRNA pairs. miRNA, microRNA.
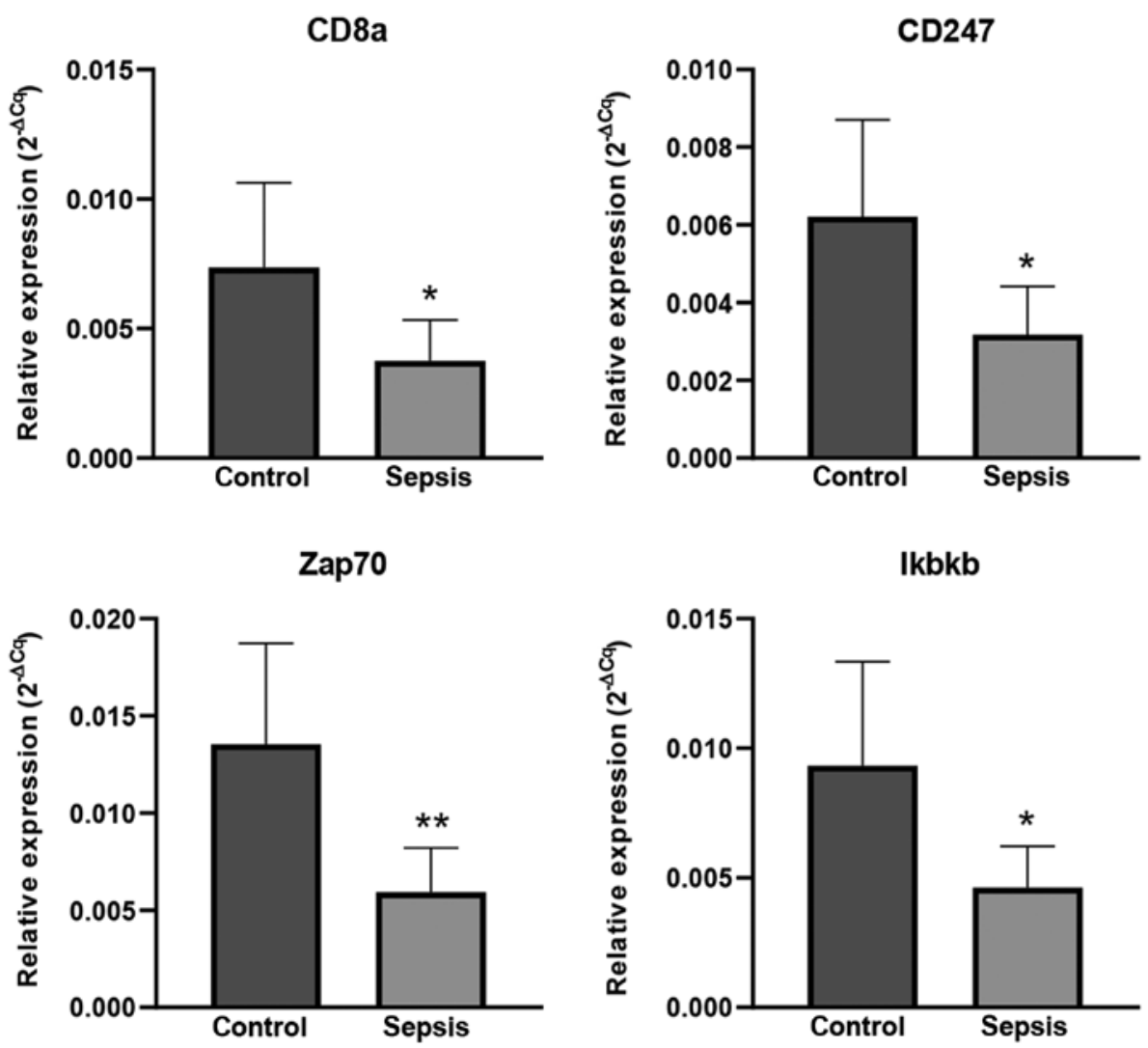

Figure 6. Validation by reverse transcription-quantitative PCR of the 4 selected mRNAs. Control indicates sham group samples. Sepsis indicates samples of CLP group. ${ }^{*} \mathrm{P}<0.05$ and ${ }^{* *} \mathrm{P}<0.01$ vs. the control. CLP, cecal ligation and puncture; $\mathrm{CD}$, cluster of differentiation. 
have indicated that a mutation in IKBKB can lead to a lack of $\operatorname{IKK} \beta$ and IKK $\beta$ deficiency leads to an inadequate response to stimuli in immune cells, finally resulting in immune impairment $(37,38)$.

In the present study, using mRNA and miRNA microarray datasets and bioinformatics analysis, 4 sepsis-related miRNA-mRNA pairs were obtained, which were enriched in the TCR signaling pathway. The differences in the expression levels also reflect functional changes in sepsis, which may contribute to immune deficiency. These results could improve understanding of the underlying molecular events in sepsis and these candidate miRNA-mRNA pairs may be predictors of, or therapy targets for sepsis. However, there were certain limitations in the present study. Firstly, the number of samples in the two datasets was small. Secondly, the CLP model mice of the two datasets were not established by the same research group. Thirdly, mmu-miR-370-3p was not verified and the causal regulatory relationship between miRNA and mRNA was not further confirmed. This causal regulation relationship may be verified using miR-mimics, in vitro inhibitors or mutant generation and adenoviral-mediated miRNA delivery in vivo. These experiments are important for future studies.

In conclusion, using miRNA and mRNA microarray datasets and multi-step bioinformatics analysis, four significant miRNA-mRNA pairs were found in sepsis model mice. These hub genes were enriched in the TCR signaling pathway, played an important role in immunity and may be markers or therapeutic targets for sepsis.

\section{Acknowledgements}

Not applicable.

\section{Funding}

The present study was supported by the National Natural Science Foundation of China (grant. no. 81860334) and Fujian Provincial Natural Science Foundation (grant. no. 2019J01585).

\section{Availability of data and materials}

The datasets analyzed (GSE74952 and GSE55238) during the present study are available in the Gene Expression Omnibus database.

\section{Authors' contributions}

JC analyzed the microarray data, performed the experiments and was a major contributor in writing the manuscript. ML downloaded the datasets and performed statistical analysis. SZ designed the study and edited the manuscript. All authors have read and approved the final manuscript.

\section{Ethics approval and consent to participate}

The present study was approved by the Animal Care Committee of Guangxi Medical University (approval no. 201904001).

\section{Patient consent for publication}

Not applicable.

\section{Competing interests}

The authors declare that they have no competing interests.

\section{References}

1. Singer M, Deutschman CS, Seymour CW, Shankar-Hari M, Annane D, Bauer M, Bellomo R, Bernard GR, Chiche JD, Coopersmith CM, et al: The third international consensus definitions for sepsis and septic shock (Sepsis-3). JAMA 315: 801-810, 2016.

2. Cecconi M, Evans L, Levy M and Rhodes A: Sepsis and septic shock. Lancet 392: 75-87, 2018.

3. Gobatto AL, Besen BA and Azevedo LC: How can we estimate sepsis incidence and mortality? Shock 47 (1S Suppl 1): S6-S11, 2017.

4. Martin GS: Sepsis, severe sepsis and septic shock: Changes in incidence, pathogens and outcomes. Expert Rev Anti Infect Ther 10: 701-706, 2012.

5. Mayr FB, Yende S and Angus DC: Epidemiology of severe sepsis. Virulence 5: 4-11, 2014.

6 . Vincent JL: Individual gene expression and personalised medicine in sepsis. Lancet Respir Med 4: 242-243, 2016.

7. Maslove DM and Wong HR: Gene expression profiling in sepsis: Timing, tissue, and translational considerations. Trends Mol Med 20: 204-213, 2014.

8. Ullah S, John P and Bhatti A: MicroRNAs with a role in gene regulation and in human diseases. Mol Biol Rep 41: 225-232, 2014.

9. Eledge MR and Yeruva L: Host and pathogen interface: MicroRNAs are modulators of disease outcome. Microbes Infect 20: 410-415, 2018.

10. Chen J, Jiang S, Cao Y and Yang Y: Altered miRNAs expression profiles and modulation of immune response genes and proteins during neonatal sepsis. J Clin Immunol 34: 340-348, 2014.

11. Barrett T, Wilhite SE, Ledoux P, Evangelista C, Kim IF, Tomashevsky M, Marshall KA, Phillippy KH, Sherman PM, Holko M, et al: NCBI GEO: Archive for functional genomics data sets-update. Nucleic Acids Res 41: D991-D995, 2013.

12. Rittirsch D, Huber-Lang MS, Flierl MA and Ward PA: Immunodesign of experimental sepsis by cecal ligation and puncture. Nat Protoc 4: 31-36, 2009.

13. Kanehisa M, Sato Y, Furumichi M, Morishima K and Tanabe M: New approach for understanding genome variations in KEGG. Nucleic Acids Res 47: D590-D595, 2019.

14. Schmittgen TD and Livak KJ: Analyzing real-time PCR data by the comparative C(T) method. Nat Protoc 3: 1101-1108, 2008.

15. Borken F, Markwart R, Requardt RP, Schubert K, Spacek M, Verner M, Rückriem S, Scherag A, Oehmichen F, Brunkhorst FM and Rubio I: Chronic critical illness from sepsis is associated with an enhanced TCR response. J Immunol 198: 4781-4791, 2017.

16. Patil NK, Bohannon JK, Luan L, Guo Y, Fensterheim B, Hernandez A, Wang J and Sherwood ER: Flt3 ligand treatment attenuates $\mathrm{T}$ cell dysfunction and improves survival in a murine model of burn wound sepsis. Shock 47: 40-51, 2017.

17. Ramonell KM, Zhang W, Hadley A, Chen CW, Fay KT, Lyons JD, Klingensmith NJ, McConnell KW, Coopersmith CM and Ford M: CXCR4 blockade decreases CD4+ T cell exhaustion and improves survival in a murine model of polymicrobial sepsis. PLoS One 12: e0188882, 2017.

18. Oami T, Watanabe E, Hatano M, Sunahara S, Fujimura L, Sakamoto A, Ito C, Toshimori K and Oda S: Suppression of $\mathrm{T}$ cell autophagy results in decreased viability and function of $\mathrm{T}$ cells through accelerated apoptosis in a murine sepsis model. Crit Care Med 45: e77-e85, 2017.

19. Cao C, Chai Y, Shou S, Wang J, Huang Y and Ma T: Toll-like receptor 4 deficiency increases resistance in sepsis-induced immune dysfunction. Int Immunopharmacol 54: 169-176, 2018.

20. Condotta SA, Khan SH, Rai D, Griffith TS and Badovinac VP: Polymicrobial sepsis increases susceptibility to chronic viral infection and exacerbates CD ${ }^{+} \mathrm{T}$ cell exhaustion. J Immunol 195: $116-125,2015$ 
21. Hotchkiss RS, Tinsley KW, Swanson PE, Schmieg RE Jr, Hui JJ, Chang KC, Osborne DF, Freeman BD, Cobb JP, Buchman TG and Karl IE: Sepsis-induced apoptosis causes progressive profound depletion of B and CD4+ T lymphocytes in humans. J Immunol 166: 6952-6963, 2001.

22. van der Poll T, van de Veerdonk FL, Scicluna BP and Netea MG: The immunopathology of sepsis and potential therapeutic targets. Nat Rev Immunol 17: 407-420, 2017.

23. Boomer JS, To K, Chang KC, Takasu O, Osborne DF, Walton AH, Bricker TL, Jarman SD II, Kreisel D, Krupnick AS, et al: Immunosuppression in patients who die of sepsis and multiple organ failure. JAMA 306: 2594-2605, 2011.

24. Inoue S, Suzuki K, Komori Y, Morishita Y, Suzuki-Utsunomiya K, Hozumi K, Inokuchi S and Sato T: Persistent inflammation and $\mathrm{T}$ cell exhaustion in severe sepsis in the elderly. Crit Care 18: R130, 2014.

25. Gil D, Schrum AG, Daniels MA and Palmer E: A role for CD8 in the developmental tuning of antigen recognition and CD3 conformational change. J Immunol 180: 3900-3909, 2008.

26. Harty JT, Tvinnereim AR and White DW: CD8+ T cell effector mechanisms in resistance to infection. Annu Rev Immunol 18: 275-308, 2000

27. Dumontet E, Osman J, Guillemont-Lambert N, Cros G, Moshous D and Picard C: Recurrent respiratory infections revealing CD8 $\alpha$ Deficiency. J Clin Immunol 35: 692-695, 2015.

28. Harland KL, Day EB, Apte SH, Russ BE, Doherty PC, Turner S. and Kelso A: Epigenetic plasticity of Cd8a locus during CD8(+) T-cell development and effector differentiation and reprogramming. Nat Commun 5: 3547, 2014

29. Irving BA and Weiss A: The cytoplasmic domain of the T cell receptor zeta chain is sufficient to couple to receptor-associated signal transduction pathways. Cell 64: 891-901, 1991.

30. D'Oro U, Munitic I, Chacko G, Karpova T, McNally J and Ashwell JD: Regulation of constitutive TCR internalization by the zeta-chain. J Immunol 169: 6269-6278, 2002.
31. Eldor R, Klieger Y, Sade-Feldman M, Vaknin I, Varfolomeev I, Fuchs C and Baniyash M: CD247, a novel T cell-derived diagnostic and prognostic biomarker for detecting disease progression and severity in patients with type 2 diabetes. Diabetes Care 38: 113-118, 2015.

32. Picard C, Dogniaux S, Chemin K, Maciorowski Z, Lim A, Mazerolles F, Rieux-Laucat F, Stolzenberg MC, Debre M, Magny JP, et al: Hypomorphic mutation of ZAP70 in human results in a late onset immunodeficiency and no autoimmunity. Eur J Immunol 39: 1966-1976, 2009.

33. Roifman CM, Dadi H, Somech R, Nahum A and Sharfe N Characterization of $\zeta$-associated protein, $70 \mathrm{kd}$ (ZAP70)-deficient human lymphocytes. J Allergy Clin Immunol 126: 1226-1233.e1, 2010.

34. Huang L, Qiao L, Zhu H, Jiang L and Yin L: Genomics of neonatal sepsis: Has-miR-150 targeting BCL11B functions in disease progression. Ital J Pediatr 44: 145, 2018.

35. Hayden MS and Ghosh S: Shared principles in NF-kappaB signaling. Cell 132: 344-362, 2008.

36. Perkins ND: Integrating cell-signalling pathways with NF-kappaB and IKK function. Nat Rev Mol Cell Biol 8: 49-62, 2007.

37. Pannicke U, Baumann B, Fuchs S, Henneke P, Rensing-Ehl A, Rizzi M, Janda A, Hese K, Schlesier M, Holzmann K, et al: Deficiency of innate and acquired immunity caused by an IKBKB mutation. N Engl J Med 369: 2504-2514, 2013.

38. Mousallem T, Yang J, Urban TJ, Wang H, Adeli M, Parrott RE, Roberts JL, Goldstein DB, Buckley RH and Zhong XP: A nonsense mutation in IKBKB causes combined immunodeficiency. Blood 124: 2046-2050, 2014.

(7) $\Theta$ This work is licensed under a Creative Commons

Attribution-NonCommercial-NoDerivatives 4.0 International (CC BY-NC-ND 4.0) License. 